\title{
The Applicability of a Geomagnetic Field based Positioning Technique with Mobile Phone to Underground Tunnels
}

\author{
T. Makkonen ${ }^{a}$, R. Heikkiläa ${ }^{\mathrm{a}}$ A. Kaaranka ${ }^{\mathrm{a}}$ \\ ${ }^{a}$ Construction Technology Research Center, University of Oulu, Finland \\ E-mail: Tomi.Makkonen@oulu.fi, Rauno.Heikkila@oulu.fi, Annemari.Kaaranka@oulu.fi
}

\begin{abstract}
In underground mines and tunnels accurate and continuous positioning of moving machines and humans has been studied extensively. Geomagnetic field based positioning is an interesting possibility for this measurement need. Using a commercial smart phone and a new calculation service provided by a Finnish company, a feasibility and accuracy assertion tests were done in Finnish underground mine. First time a standard mobile phone, as far as we know, was used in underground tunnel environment to produce continuous position data and an accuracy assessment was performed. An Lshaped tunnel area, two tunnels crossing, was selected for the experiments. A total station was used for reference measurements. Accuracy of measurement in different static and dynamic situations was studied.

The found RMSE of 7.32 meters should satisfy many needs. The use of standard smart phone for underground positioning worked fine, but more development work is needed it to be usable in everyday mining situations.
\end{abstract}

Keywords -

Magnetic field; Underground positioning; Tunnel

\section{Introduction}

The need of continuous positioning in underground mines is needed among other things for safety, logistics, machine operations and machine automation [1], [2]. Position technologies are widely applied to above ground level tasks mainly because GPS is so effortless to use. There has not been commercial service which could position underground effortlessly.

The best position method currently is obviously a total station and they are widely used, however they need to be set up and moved according to the positioning needs. The narrow corridors add challenge for setting up the device and also the requirement of direct view complicates measurements.

Several positioning solutions have been proposed for the underground mines, such as radio signal based positioning [3], [4] and the use of RFID [5], [4]. CSIRO has made lot of work with their Inertial Navigation system to advance the field of automated continuous mining equipment [6].

A geomagnetic field based positioning technique for the underground mines was suggested and tested by Haverinen and Kemppainen [7]. The magnetic field of Earth is not constant, but has a number of anomalies inside the buildings [8] as well as in mines when magnetic minerals are present. Measuring these anomalies and generating a magnetic field map, one can use this data to successful positioning.

Haverinen and Kemppainen made a positioning test underground with an accuracy of about 1.5 meters. They used a magnetometer sensor array at approximate length of 1 meter. [7] Their work with geomagnetic positioning technique lead to company called IndoorAtlas which has a business model to provide web based service for position calculations using Monte-Carlo Localization from recorded geomagnetic maps and tools in mobile phone to do the mapping inside the buildings. In most mobile phones there already is a three-axis magnetometer costing less than one dollar making the device a magnetometer [9].

Magnetic field positioning indoors has been studied increasingly in recent years [10]-[15]. There were not prior test with geomagnetic positioning underground using mobile phone thus the results are quite unique.

The measurements were done in the Outokumpu Kemi mine, a chromium mine producing 2.7 million tons of ore per year in northern Finland, about 500 meters below the surface.

\section{Measurements and setup}

Main tools for measurements were:

1. IndoorAtlas web service for creating and maintaining maps

2. IndoorAtlas Mobile APK for creating magnetic maps and visual testing on the site 
3. KemiMineDataAquisition APK mobile software for recording the location data

4. Android Nexus 4 phone

5. Kemi Mine wireless network

Because of the novel use of cell phone to get position data undergrounds a group tests were performed using setup seen in Figure 1. Performed tests where: A. Visual inspection of usability B. Positioning error on the central line of the tunnel, where the mapping was made in 10 separate locations. C. Positioning error on the central line of the tunnel, when walking distance was increased by including every second point. D. Positioning error on the side of the tunnel in 10 separate locations. E. Initial tests with vehicles for future use.

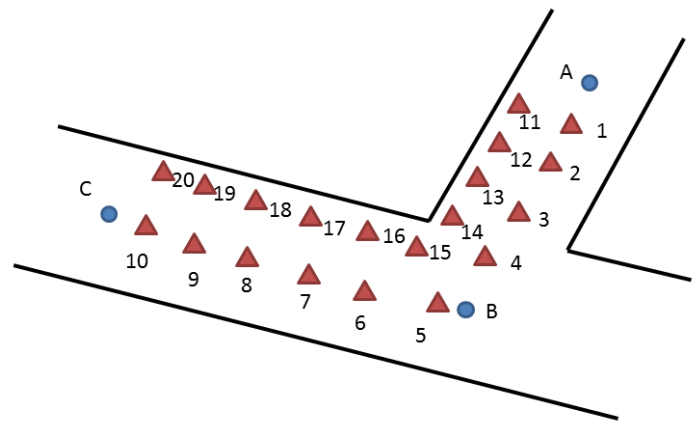

Figure 1. Schematic map about the experiment

\subsection{Principle of magnetic field based positioning}

The magnetic field is usually presented as a constant field as in Figure 2. Magnetic field has local variation as well as time based variation. Figure 3 shows a more localized map of the size of $5 \mathrm{~km} \times 5 \mathrm{~km}$, where variations are clearly seen.

Finally on the mine tunnel level (Figure 4) we see the same variations on the magnetic field. Using this data as a map a position can be calculated. Fluctuations on the magnetic field can be both natural or generated by steel and reinforced concrete structures, electric power systems, electric and electronic appliances [8]. In the mines sufficient variations in magnetic fields to enable magnetic field positioning is still a question mark as the man made magnetic constructions and local environmental variations are different around the globebut it has been shown to work now at least in two mines in Finland.

It has to be noted that the magnetic map is not unique in given location, but algorithms are used to derive position when object moves through the map. More there is anomalies the easier the location is to define [10]. Widely used algorithm in robotics to this task is Monte Carlo localization.

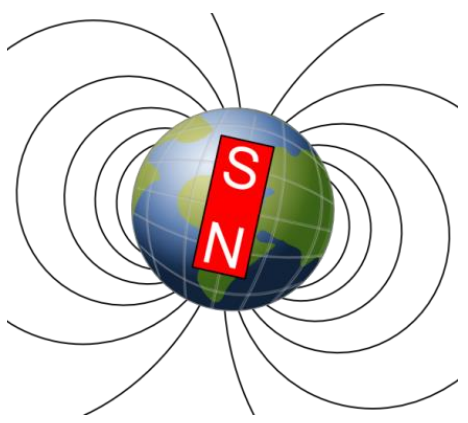

Figure 2. Earth's magnetic field. Source: Wikimedia Commons. Image by Zurek [16]

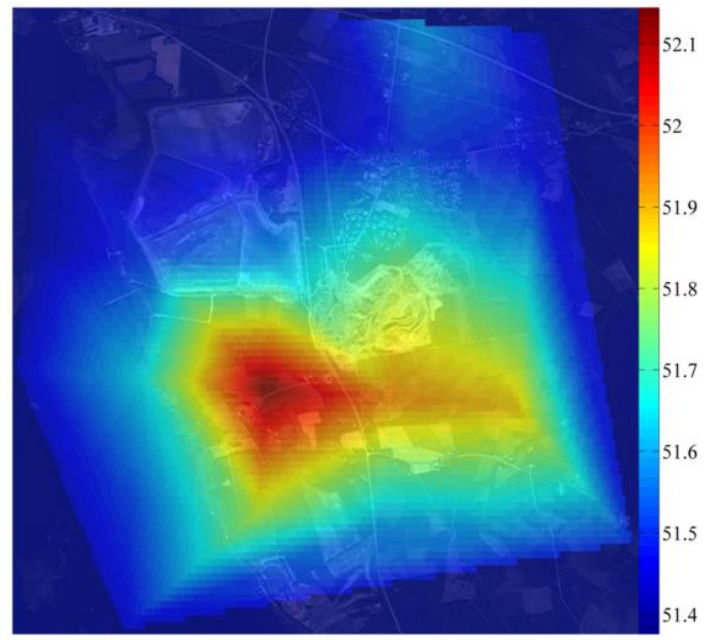

Figure 3. Magnetic map $[\mu T]$ of the Pyhäsämi mining area in Finland. Image courtesy of $\mathrm{J}$. Haverinen and A. Kemppainen [7]

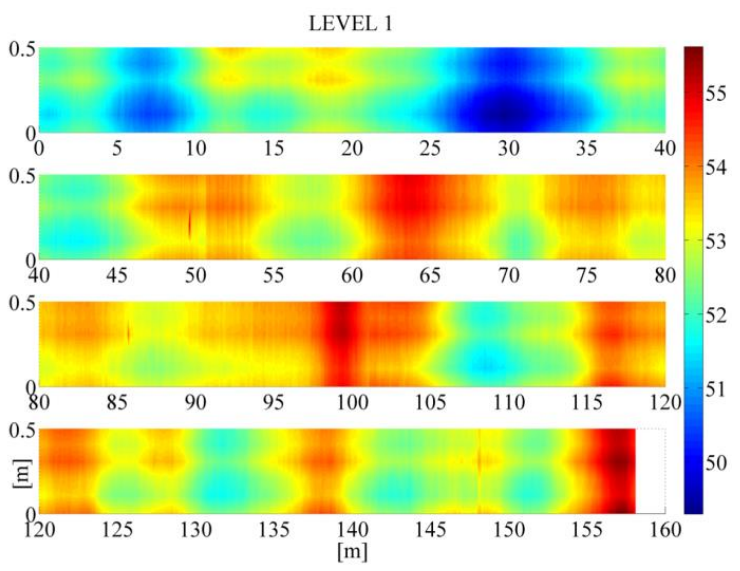

Figure 4. Magnetic field map $[\mu T]$ of the tunnel. Image courtesy of $J$. Haverinen and $A$. Kemppainen [7] 


\subsection{Data acquisition preparations}

Pre data acquisition a map of the studied tunnel was imported to the IndoorAtlas service. Three points from the map were chosen as wide apart as possible (600 meters) and were mapped to correspond to known locations. Points in the picture were chosen as carefully as possible using image viewer with full zoom to get $x y$ pixel coordinates. Then these points were referenced to known reference coordinate points (EUREF89) using the IndoorAtlas web service, see Figure5. The image was automatically resized when moved to the cloud by the web service and thus scaling with image size was calculated to three reference picture points.

Two sources of error are present here. Firstly, selecting the correct pixels contains an error and secondly, reference points are transformed from mine specific coordinate system to KKJ (an older Finnish standard) and then again to EUREF89. Further the IndoorAtlas uses WGS84-coordinate system and there is an error element between the two of magnitude of 30 $\mathrm{cm}$ in the year 2009 [17].

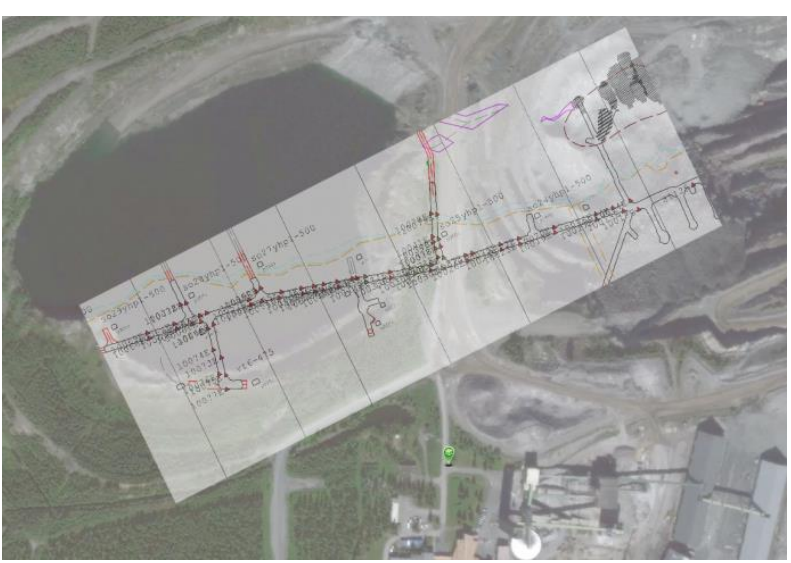

Figure 5. A map of the tunnel used in the measurements as seen in the IndoorAtlas web service. Three reference points to fix map to correct location was used.

A program, KemiMineDataMain, was created to Android phone by modifying IndoorAtlas API Example 0.53, see Figure 6. Data saving, time stamp and measurement running index were added. The programming language JAVA was used with Eclipse IDE. The program will list current time, number of measurement, roundtrip time to the servers, Lat and Lon in WGS84, $\mathrm{X}$ and $\mathrm{Y}$ in meters from the map's upper corner, I and J pixel coordinates again from the map's upper corner, heading in degrees, and server side estimation of probability about the correctness of the position.

On the site initial mapping was performed using
Android 4.4.2 LG Nexus 4 phone and software IndoorAtlas Mobile 0.6.2.1382 APK. Figure 1. shows the area mapped. The area was divided to two lines AB$\mathrm{BC}$ and magnetic field recording and validation was made.

Two lines of reference points were measured. Middle line was as close of the recorded and validated line (Figure 1) as possible and the second reference line was measured to the very side of the tunnel, of where we assume the largest errors are due the measurement and validation method.

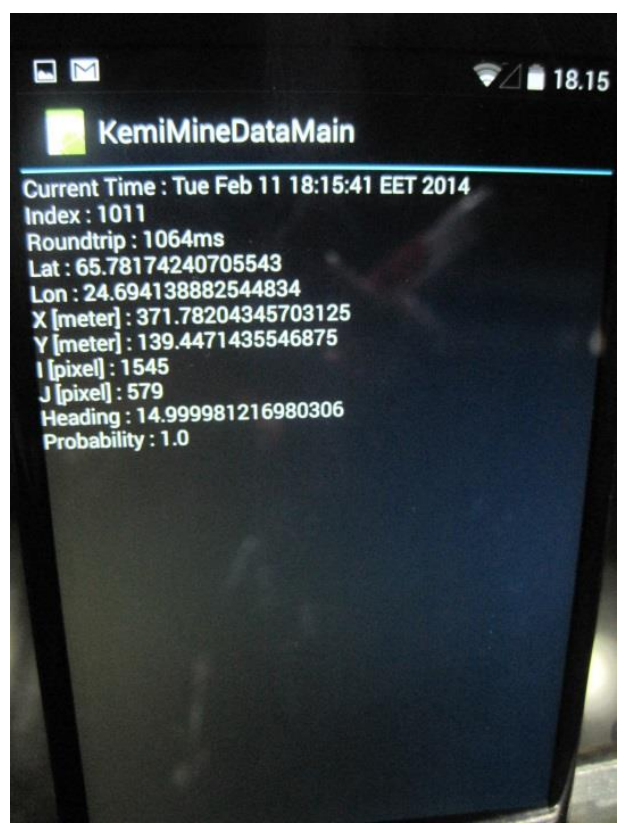

Figure 6. KemiMineDataMain. Android data collection software running on Nexus 4 phone.

\subsubsection{Measurement A}

Visual inspection of usability was performed using IndoorAtlas Mobile APK where researchers and mine worker walked around the mapped area and made their observations (Figure 9). In this experiment a map of the area was presented and a person's location was moving just like it does in everyday GPS-based localization software.

\subsubsection{Measurement B}

Positioning error on the central line of the tunnel was measured next. Ten reference points were measured with Leica Viva R1000 robotic total station (Figure 8.) created in an L-shape (Figure 1) with 4 points in the cross tunnel and 6 in the main tunnel. These points were measured in the Kemi mine coordinate system. A total of 40 measurements were made, every point four times, 
along the path 1 to 10 - 10 to 1 - 1 to 10 - 10 to 1 . First half of the data measurements were made using statiivi so that the phone stays totally still for one minute. After realizing the results do not change if the movement of the phone is small, the rest 20 points were measured keeping the phone still by hands only the time that was need to take down the measurements.

\subsubsection{Measurement C}

Positioning error on the central line with increased walking distance was performed using the same 10 reference points than in measurement $\mathrm{B}$. The distance was increased because we made an assumption that system will perform better this way due its location principles. A total of 20 measurements were made in order (see Figure 1.) 10-8-6-4-2-1-3-5-7-9.

\subsubsection{Measurement D}

Positioning error on the side of the tunnel was performed along the path 11 to 20, see Figure 1. The points were measured as close to the tunnel wall as reasonable. Procedure was same than what was used in the Measurement B. A total of 10 reference points were used with two measurement each.

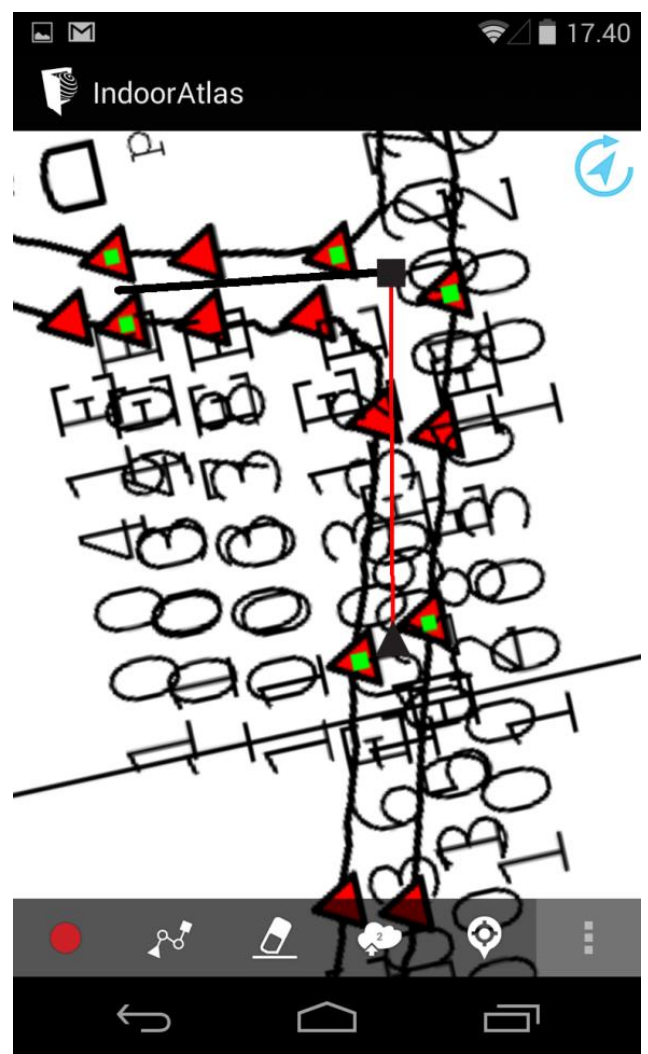

Figure 7. Mapped area in the tunnel. In the centre of the tunnel red line is recorded and black line is currently validated.

\subsubsection{Measurement E}

Vehicle tests were performed as an initial preparation for full tests in the future. A magnetic field magnitude was measured at the distances of $15 \mathrm{~m}, 10 \mathrm{~m}$, $5 \mathrm{~m}$ and $0 \mathrm{~m}$ away from the operational mining drill (in Figure 8.) using mobile application on Iphone4, with ten measurements on each point. Then a magnetic field was measured using the same application when mine van car drove from the 10 meters to $10 \mathrm{~cm}$ from the device and also by visually checking the position data. Also xycoordinates were recorded 3 times when the mine van car drove from about 5 meters to the close proximity of magnetic field positioning device.

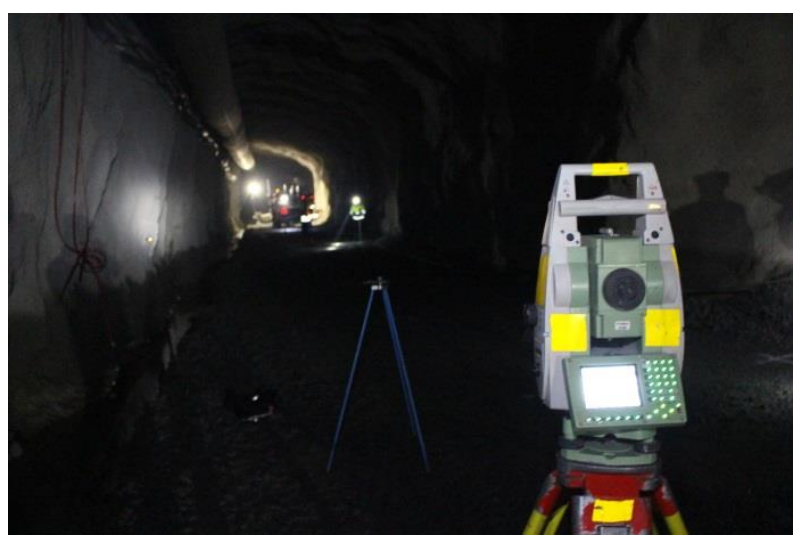

Figure 8. Total station and mine drill used on the measurement $\mathrm{E}$.

\subsection{Coordinate transformations and error analysis}

Error calculations were performed in the Kemi mine coordinate system (KMCS) and are presented in meters. The procedure to calculate errors was same in Measurements B,C and D.

For calculating coordinate transformation to $\mathrm{KMCS}$ from WGS84 three known points were used as far apart as possible on the mine map (Figure 5) and mapping was done using six parameter affine transformation, further, ETRS89 and WGS84 were treated as a same coordinate system. Kemi Mine uses wide net of reference points for total stations which are known in KMCS and KKJ. These points were used to check the calculated transformation with the standard transformation KKJ to ETRS89.

Measurement reference points (Figure 1) are known in KMCS, but measured positions with the phone are acquired in WGS84. Again using assumption given earlier and a six parameter affine transformation a transformation chain WGS84-ETRS89-KMCS is ready. 
These values are then compared to the values measured with a total station in the KMCS.

Root-mean-square-errors (RMSE) were calculated, Equation (1) where $\hat{x}_{t}$ is predicted value and $x_{t}$ reference value.

$$
R M S E=\sqrt{\frac{\sum_{t=1}^{n}\left(\hat{x}_{t}-x_{t}\right)^{2}}{n}}
$$

Average error, also in this case know as mean bias error (MBE) [18], is calculated using equation 2.

$$
M B E=\frac{\sum_{i=1}^{n}\left(\hat{x}_{i}-x_{i}\right)}{n}
$$

For precision evaluation standard deviation is calculated.

Because mapping was done on the central line of the tunnel, the results on the Measurement $D$ will suffer a bias as the measurements are projected to this mapped line. There seems to be no difference in Measurement $B$ and $\mathrm{C}$, thus combining these measurements for a larger measurement group is used as a main data set to study error.

Additionally errors for distance $d$ from $\Delta x$ and $\Delta y$ was calculated, defined in Equation (3).

$$
d=\sqrt{\Delta x^{2}+\Delta y^{2}}
$$

For errors in vehicle test average magnitude was used when measurements were made. The visual inspections were performed as subjective opinions.

\section{Results}

Visual inspection of the usability of the geomagnetic field based positioning technique with mobile phone 500 meters underground gave good results. The position presented graphically in the screen of the smart phone was deemed satisfactory for positioning a person underground by all four testers. In Figure 9 a positioning view is shown, where position of the phone is shown inside a blue circle. Notice how predicted position circle gets smaller, when convergence error is estimated to get smaller.

Root-mean-square error was calculated for Measurements $B, C, D$ and combined $B+C$ and also total distance error $d$ (Table 1). Other measurements include: standard deviation (Table 2), Maximum errors (Table 3) and MBE (Table 4)
Table 1. Calculated RMSE values

\begin{tabular}{cccc}
\hline Measurement & $\mathrm{x}(\mathrm{m})$ & $\mathrm{y}(\mathrm{m})$ & $d(\mathrm{~m})$ \\
\hline $\mathrm{B}$ & 6.27 & 3.12 & 7.00 \\
$\mathrm{C}$ & 7.51 & 2.47 & 7.90 \\
$\mathrm{~B}+\mathrm{C}$ & 6.71 & 2.92 & 7.32 \\
$\mathrm{D}$ & 8.83 & 3.56 & 9.52 \\
\hline
\end{tabular}

Table 2. Calcualted standard deviation

\begin{tabular}{cccc}
\hline Measurement & $\mathrm{x}(\mathrm{m})$ & $\mathrm{y}(\mathrm{m})$ & $d(m)$ \\
\hline B & 4.88 & 2.46 & 4.23 \\
C & 4.57 & 1.47 & 4.32 \\
B+C & 6.70 & 2.89 & 4.22 \\
D & 4.57 & 1.47 & 4.32 \\
\hline
\end{tabular}

Table 3. Calculated maximum errors

\begin{tabular}{cccc}
\hline Measurement & $\mathrm{x}(\mathrm{m})$ & $\mathrm{y}(\mathrm{m})$ & $d(m)$ \\
\hline B & 19.98 & 7.20 & 20.62 \\
C & 19.15 & 4.84 & 19.48 \\
B+C & 19.97 & 7.20 & 20.62 \\
D & 16.45 & 5.01 & 17.20 \\
\hline
\end{tabular}

Table 4. Calculated MBE

\begin{tabular}{clll}
\hline Measurement & $\mathrm{x}(\mathrm{m})$ & $\mathrm{y}(\mathrm{m})$ & $d(m)$ \\
\hline $\mathrm{B}$ & 3.39 & 3.09 & 4.17 \\
$\mathrm{C}$ & 4.88 & 2.46 & 4.23 \\
$\mathrm{~B}+\mathrm{C}$ & 0.36 & 0.36 & 5.98 \\
$\mathrm{D}$ & -7.56 & 3.25 & 8.48 \\
\hline
\end{tabular}

The transformation error on the measurement area between KMCS and WGS84 was on average $0.04 \mathrm{~cm}$ and $-2.58 \mathrm{~cm}$ for $\mathrm{x}$ and $\mathrm{y}$ directions, with standard deviation $0.03 \mathrm{~cm}$ and $0.21 \mathrm{~cm}$.

The averages for magnetic field magnitudes when approaching drilling machine were: $54.2 \mu \mathrm{T}(15 \mathrm{~m})$, $49.6 \mu \mathrm{T}(10 \mathrm{~m}), 53.7 \mu \mathrm{T}(5 \mathrm{~m}$, another vehicle close $)$ and $44.3 \mu \mathrm{T}(0 \mathrm{~m})$.

Mine van approaching gave average errors $1.73 \mathrm{~m}$ and $1.06 \mathrm{~m}$, for $\mathrm{x}$ and $\mathrm{y}$ directions.

\section{Conclusion}

The experiments and measurements for magnetic field based positioning error using mobile phone showed great promise. Talks with the Kemi mine staff revealed that first encounter with this positioning technique was pleasant. RMSE value of $7.32 \mathrm{~m}$ on the central line of the tunnel was calculated and for unmapped area (side of the tunnel) $9.52 \mathrm{~m}$. The standard deviation behaved nicely between 4.22 - 4.23 meters between the different measurements. This accuracy is 
enough for safety and logistic operations. However, when we compare the accuracy to earlier work [7] we see a quite large difference of almost 6 meters. The obvious difference between the two is the measurement device, in this measurement was done using standard smart phone, in earlier work a specially designed sensor array was used.

The max distance error is quite large and reason for that is unknown, it can be a glitch or Monte-Carlo Localization was performing badly due magnetic field being too monotone or a convergence error is possible too. Also one possible source for it is a break in a data connection.

The vehicle experiments showed also great promise to the future work. There seemed to be no problems when vehicle was near, although we do have to remember that these tests were not performed in-depth enough to answer if vehicles are a problem to the method. There seem to be some changes in the magnetic field near the vehicles.

To work the measurement device needs a constant internet access; this is possible in the Kemi mine but not necessary in all other mines. To make system work in all possible scenarios underground we feel that the magnetic field positioning calculations should be performed on the phone. This also would make system safer for disturbances like fire and landslide not to mention everyday problems every wireless network goes through time to time.

The positioning system is also, at the moment, only optimized for people walking. When the distances are great, like in mines, this is not convenient. However, when it's now proven that geomagnetic positioning with mobile phone works underground, there shouldn't be any technical or financial obstacles to overcome this limitation. There could be for example a fixed unit on the vehicle completed with odometry.

Currently there is no support for automatic floor selection, this could be solved in future versions and should be solvable already by combining positioning techniques like wireless base station selection for the map.

We hope that this positioning technique will be seen in mines in the near future in daily usage.

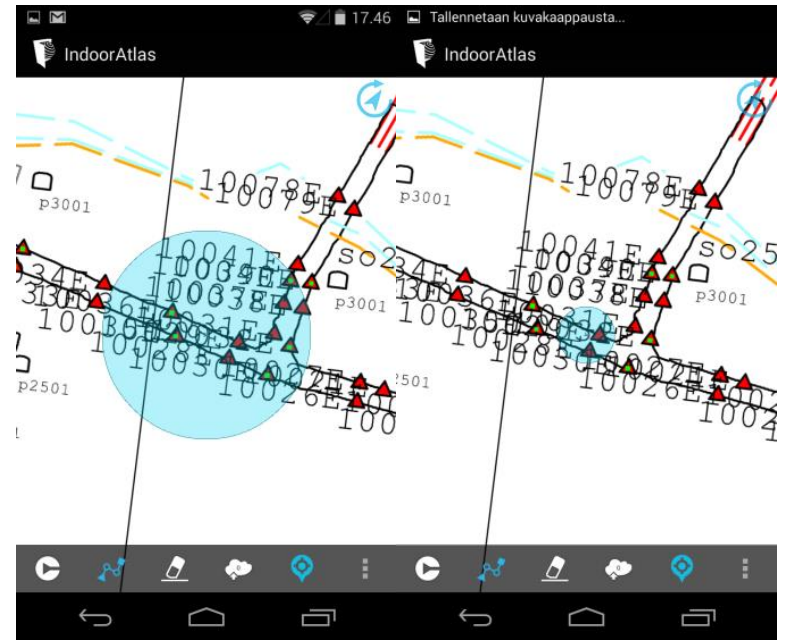

Figure 9. Visual inspection of the positioning.

\section{References}

[1] S. Zhong, Proceedings of the 2012 International Conference on Cybernetics and Informatics. Springer, 2013.

[2] S. van Duin, L. Meers, and G. Gibson, "HARD AUTOMATION TRENDS IN AUSTRALIAN UNDERGROUND COAL MINES," in 30th International Symposium of Automation and Robotics in Construction and Mining (ISARC 2013) Proceedings, 2013.

[3] X. Huang, W. Zhu, and D. Lu, "Underground miners localization system based on ZigBee and WebGIS," in 2010 18th International Conference on Geoinformatics, 2010, pp. 1-5.

[4] L. Zhang, X. Li, L. Chen, S. Yu, and N. Xiao, "Localization system of underground mine trackless facilities based on Wireless Sensor Networks," in IEEE International Conference on Mechatronics and Automation, 2008. ICMA 2008, 2008, pp. 347-351.

[5] A. F. C. Errington, B. L. F. Daku, and A. F. Prugger, "Initial Position Estimation Using RFID Tags: A Least-Squares Approach," IEEE Trans. Instrum. Meas., vol. 59, no. 11, pp. 2863-2869, Mar. 2010.

[6] M. Dunn, P. Reid, and J. Ralston, "A practical inertial navigation solution for continuous miner automation," Coal Oper. Conf., Jan. 2012.

[7] J. Haverinen and A. Kemppainen, “A geomagnetic field based positioning technique for 
underground mines," in 2011 IEEE International Symposium on Robotic and Sensors Environments (ROSE), 2011, pp. 7-12.

[8] J. Haverinen and A. Kemppainen, "Global indoor self-localization based on the ambient magnetic field," Robot. Auton. Syst., vol. 57, no. 10, pp. 1028 1035, Oct. 2009.

[9] “A Compass in Every Smartphone - IEEE Spectrum." [Online]. Available: http://spectrum.ieee.org/semiconductors/devices/acompass-in-every-smartphone. [Accessed: 14-Feb2014].

[10] B. Li, T. Gallagher, A. G. Dempster, and C. Rizos, "How feasible is the use of magnetic field alone for indoor positioning?," in 2012 International Conference on Indoor Positioning and Indoor Navigation (IPIN), 2012, pp. 1-9.

[11] J. Chung, M. Donahoe, C. Schmandt, I.-J. Kim, P. Razavai, and M. Wiseman, "Indoor Location Sensing Using Geo-magnetism," in Proceedings of the 9th International Conference on Mobile Systems, Applications, and Services, New York, NY, USA, 2011, pp. 141-154.

[12] S. Suksakulchai, S. Thongchai, D. M. Wilkes, and K. Kawamura, "Mobile robot localization using an electronic compass for corridor environment," in 2000 IEEE International Conference on Systems, Man, and Cybernetics, 2000, vol. 5, pp. 3354-3359 vol.5.

[13] J. Haverinen and A. Kemppainen, "A global self-localization technique utilizing local anomalies of the ambient magnetic field," in IEEE International Conference on Robotics and Automation, 2009. ICRA '09, 2009, pp. 3142-3147.

[14] D. Navarro and G. Benet, "Magnetic map building for mobile robot localization purpose," in IEEE Conference on Emerging Technologies Factory Automation, 2009. ETFA 2009, 2009, pp. 1-4.

[15] E. Georgiou and J. Dai, "Self-localization of an autonomous maneuverable nonholonomic mobile robot using a hybrid double-compass configuration," in 2010 7th International Symposium on Mechatronics and its Applications (ISMA), 2010, pp. 1-8.

[16] Zureks, "English: Schematic representation of Earth's magnetic field lines," 12-Jan-2012. [Online]. Available:

http://commons.wikimedia.org/wiki/File:Earth\%27s_ma gnetic_field,_schematic.png. [Accessed: 27-May-2014].

[17] K. Grinderud, H. Rasmussen, and S. Nilsen, GIS: The Geographic Language of Our Age. Tapir Academic Press, 2009.
[18] T. A. Reddy, Applied Data Analysis and Modeling for Energy Engineers and Scientists. Springer, 2011. 